\section{Loss of Epas1 promotes FSGS}

The mechanisms that cause focal segmental glomerulosclerosis (FSGS) are complex and only partially understood. FSGS lesions are often observed in patients with hypertension, but the mechanisms that link high blood pressure to glomerular lesions are unclear. A new study now shows that endothelial Epas1 deficiency alters local renal haemodynamics in response to the vasoconstrictor angiotensin II, leading to glomerular hypertrophy and other hallmarks of FSGS. These findings not only identify a protective role of EPAS1 during glomerular hypertensive injury but also demonstrate that endothelial signalling can trigger FSGS.

Pierre-Louis Tharaux and colleagues chose the endothelial transcription factor EPAS1 from a bioinformatic analysis that aimed to identify disease modifiers that affect susceptibility to renal injury. "Abrogation of the Epas1 gene in the endothelium was then a logical next step to test the implication of this transcription factor as a potent modulator of glomerular diseases," explains Tharaux. In response to angiotensin II infusion, mice with endothelial EPAS1 deficiency developed a similar hypertensive response to that of angiotensin II-infused control mice, but demonstrated more severe glomerulosclerosis, with progressive loss of podocytes and notable recruitment of parietal epithelial cells (PECs). "This finding was unexpected, because we did not target podocytes or the glomerular PECs directly, illustrating a role for the EPAS1 pathway in intercellular pathogenic crosstalk between the endothelium and these cell types," says Tharaux.

The researchers do not know whether this intercellular crosstalk results from alterations in glomerular haemodynamics or changes in cell-cell communication. "We plan to examine EPAS1-dependent and EPAS1-independent endothelial paracrine factors that may signal to podocytes and PECs, as well as identify regulators of the EPAS1 pathway in health and disease," concludes Tharaux.

Jack M. Heintze endothelial

Epas 1 deficiency alters local renal haemodynamics in response to the vasoconstrictor angiotensin II, leading to ... hallmarks of FSGS 